\title{
Aerodynamic Test Results of Bicycle Helmets in Different Configurations: Towards a Responsive Design
}

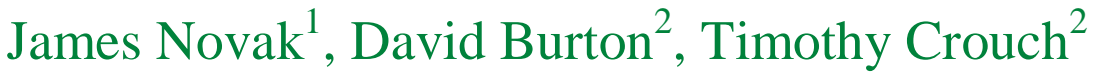 \\ ${ }^{1}$ Product Design, University of Technology Sydney, Australia \\ ${ }^{2}$ Mechanical and Aerospace Engineering, Monash University, Australia
}

\section{Corresponding Author:}

James Novak

Product Design, School of Design, Architecture and Building, University of Technology Sydney, 15 Broadway, Ultimo NSW 2007, Australia

Email: James.Novak@uts.edu.au

\section{Abstract}

Within the sport of cycling, aerodynamic efficiency is a fundamental criterion for equipment such as bicycle frames, wheels, clothing and helmets. Emerging technologies continually challenge the rules governing the sport, as designers, engineers, sports scientists and athletes attempt to gain the edge on their competition. This study compares the aerodynamic drag force of three 3D-printed bicycle helmet prototypes with three commercially available helmets via aerodynamic testing in a wind tunnel. One 3D printed helmet featured a mechanical mechanism, allowing two states of ventilation to be examined for aerodynamic efficiency, while another featured electronically adjustable ventilation tested at five different states of ventilation opening. A third 3D printed helmet acted as a control, based on a budget-level helmet design. Data was collected using an anthropometrically accurate mannequin sitting atop a bicycle in a road cycling position. The results found that the mechanically controlled prototype offered a $4.1 \%$ increase in overall drag experienced by the mannequin with ventilation in the open position compared to the closed position. The electronic prototype showed an increase in drag as ventilation opening increased through the five states, with an overall difference in drag of $3.7 \%$ between closed and the maximum opening. These experimental findings indicate the significant effect that helmet ventilation design can play on the drag forces experienced by a cyclist. This may provide new opportunities to modify athlete performance throughout varying stages of training and competition using sensors and autonomous control systems.

\section{Keywords}

3D printing, 4D product, aerodynamics, bicycle helmets, cycling, ubiquitous computing, wearable technology, wind tunnel 


\section{Introduction}

The modern sports industry is heavily influenced by technology, ${ }^{1}$ with cycling having been described by nineteenth century French author Louis Baudry de Saunier as a sport where man is "half made of flesh and half of steel that only our century of science and iron could have spawned." It is no wonder then that numerous studies have investigated the aerodynamic properties of bicycle helmets, being one of the required protective devices worn in competition under Union Cycliste Internationale (UCI) regulations, and mandatory for recreation in several countries. Studies have found that the helmet alone is responsible for $2-8 \%$ of the total aerodynamic drag on a cyclist at speeds of $30 \mathrm{~km} / \mathrm{h}$ or greater. ${ }^{3,4}$ More specific studies into the design features of specialty time-trial helmets have shown that helmet aerodynamic efficiency can be improved when time-trial helmets are designed with a long length and smooth vents. "Therefore, an aerodynamically efficient helmet can provide a competitive advantage and by selecting appropriate helmets and maintaining correct body position, a cyclist can reduce aerodynamic drag notably and the conserved energy can be used at appropriate stages of racing.,"5

A previous study by Alam et al. ${ }^{6}$ experimented with covering air vents of a Giro Atmos helmet to compare the aerodynamic and thermal changes in a wind tunnel. The results showed a $12 \%$ reduction in the drag coefficient for the modified helmet compared to the standard helmet. However, when air vents were covered, thermal performance was compromised with an increase of $1.2^{\circ} \mathrm{C}$ at a speed of $30 \mathrm{~km} / \mathrm{h}$, meaning less cooling effect for the cyclist. This study highlighted the significance that air vent location, size and quantity can have on the performance of a cyclist, important considerations cyclists need to make when selecting a helmet for competition or leisure.

A number of new bicycle helmets have emerged in recent years that give cyclists the opportunity to control this balance between aerodynamics and thermal regulation. One example is the Infinity helmet (Kask, Chiuduno, BG, Italy), which includes an adjustable ventilation piece whereby the cyclist can manually open or close the primary ventilation holes to suit their needs. For example, the vents can be opened when climbing uphill to maximize cooling or closed when sprinting to minimize drag. Similarly, the Star Pro helmet (Bell Sports, Rantoul, IL, USA) allows riders to manually control covers for the ventilation holes with a slider button. While the study by Alam et al. ${ }^{6}$ would indicate the benefits of such adaptability, peer-reviewed data has not been published about the efficiency of these commercial designs. Furthermore, the manual process of modifying ventilation in these designs is subject to human error (i.e. a cyclist may forget to open or close the vents, thereby experiencing increased aerodynamic drag or a reduced cooling effect). Furthermore, the adjustment of the vents requires the cyclist to remove their hand from the handlebar, sacrificing bicycle control for a short time.

To improve the practical implementation of adjustable vent designs, prototype helmets featuring electro-mechanical systems have been developed for this pilot study, leveraging ubiquitous computing principles (the widespread embedding of computational power and sensors into everyday objects). ${ }^{7}$ More specifically, the prototypes used in this study are examples of $4 D$ products, ${ }^{8}$ an emerging field of product development which provides the "ability for the product to physically evolve over time to suit changes in user needs," 
without direct input or control by the user. Such products may also be described as being responsive.

The primary aim of this study was to gather empirical data regarding the aerodynamic drag properties of the $3 \mathrm{D}$ printed prototype helmets with variable ventilation, comparing them to existing commercially available helmets in a number of configurations. Unlike previous studies, prototype helmets were specifically designed and 3D printed to explore variable ventilation, rather than simply taping over vents on existing designs. The secondary aim of this study was to extrapolate results into an understanding of how a responsive helmet may affect a cyclist during training or competition, allowing for further research directions beyond the scope of this study.

\section{Experimental Procedure}

\section{Description of Helmets}

This study used three commercially available bicycle helmets and three 3D printed prototype helmets as shown in Fig. 1. The S-Works Evade (Specialized Bicycle Components, Morgan Hill, CA, USA) was selected as the baseline helmet for comparison since it is a commonly available helmet widely used by recreational and professional cyclists for its aerodynamic and thermal properties. The Bambino (Kask, Chiuduno, BG, Italy) and Advantage (Giro, Santa Cruz, CA, USA) were selected as premium aerodynamic helmets typically used for time-trial racing. Using these helmets allowed the authors to gain a better understanding about aerodynamic drag at the specialist end of bicycle helmet design, while also determining whether the 3D printed prototypes could achieve similar levels of aerodynamic efficiency when vents were closed. The Bambino and Advantage were both tested with and without their visor attachments, while the Advantage was also tested with the vents taped over with the visor attached as shown in Fig. 2. 

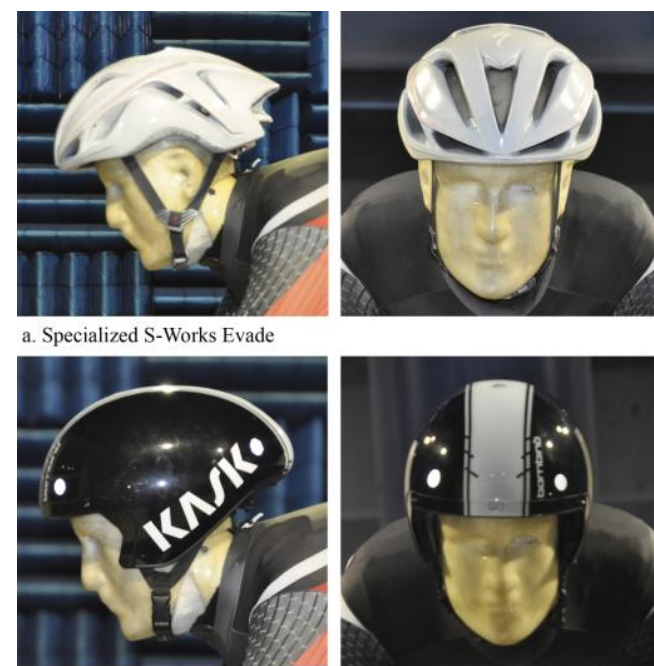

b. Kask Bambino - tested with visor on and off
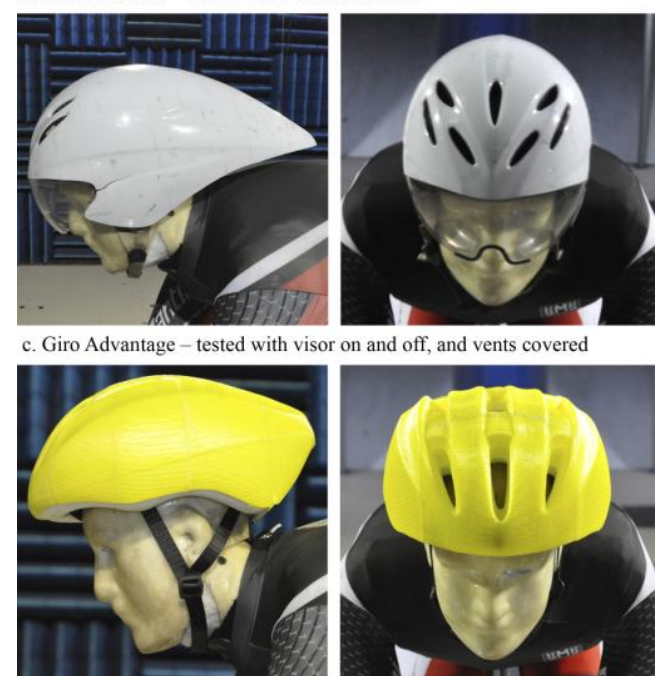

d. Prototype 1 - Passive Vents

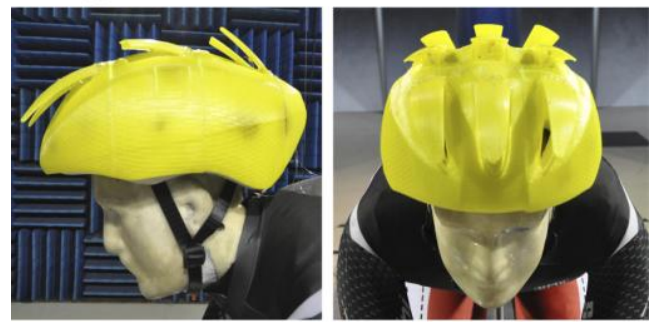

e. Prototype 2-Active Vents - tested in open and closed positions

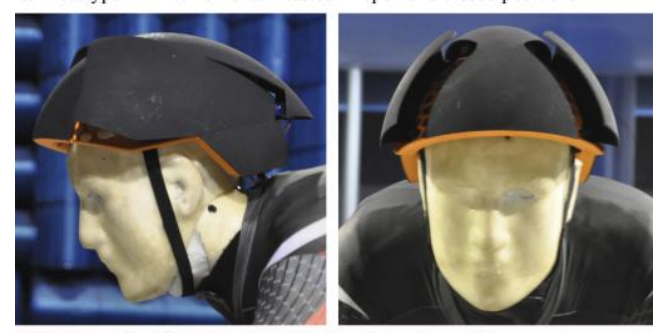

f. Prototype 3 - Dynaero - tested at 5 vent opening settings

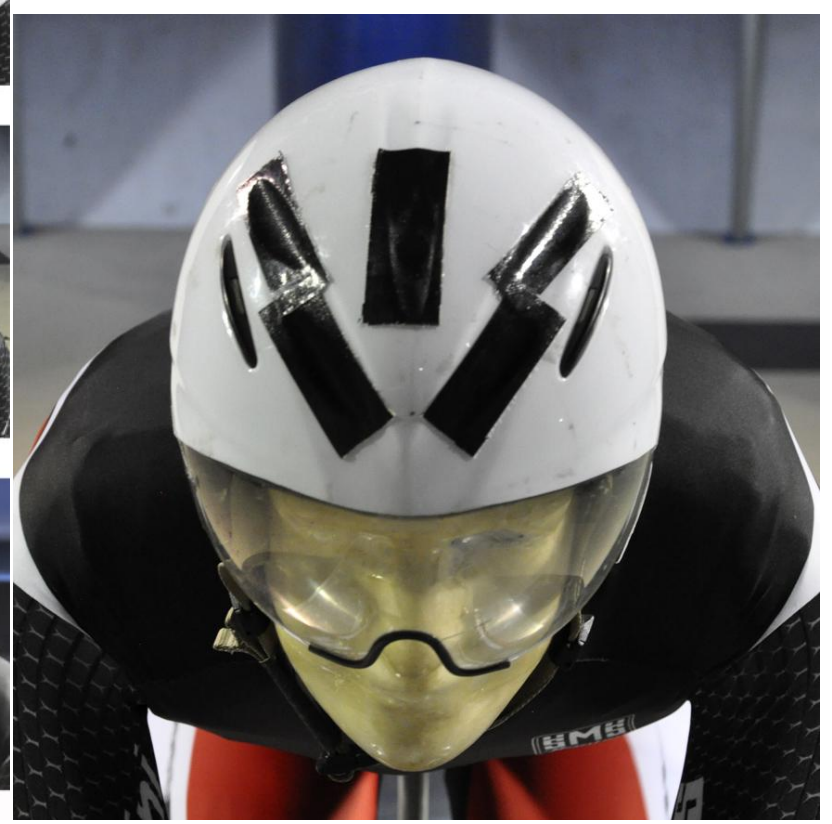

Figure 1. Front and side profiles of helmets in this study

Figure 2. Giro Advantage with vents taped and visor attached 


\section{Development of Prototypes}

The form of Prototype 1 was created by 3D scanning a budget level Series 1 helmet (Cyclops, Tullamarine, VIC, Australia), which meets Australian Standards AS/NZS 2063. The standard vacuum-formed exterior was removed and a larger 3D printed shell was produced on a standard desktop Fused-Deposition Modeling (FDM) 3D printer in multiple pieces, and then glued onto the foam interior. Prototype 2 is identical in size and shape to Prototype 1 with the same size and location of ventilation with the only difference being that Prototype 2 had additional ventilation covers, which could be opened and closed mechanically. For this study, only the open and closed positions were tested with no electronic system attached to this prototype.

Prototype 3, also known as the 'Dynaero' helmet, ${ }^{8}$ was designed as an original piece for this research and 3D printed using Selective-Laser Sintering (SLS) technology, which is more robust and accurate than FDM. This helmet has a built-in micro servo to control the opening angle of the two large vent openings, which have been designed using a different method of mechanical movement to Prototype 2 to compare the effect on aerodynamic performance in these tests. An overview of dimensions of the three 3D printed prototypes is shown in Fig. 3. A specific mobile application was developed for Android devices to control the opening of the vents of Prototype 3 via a Bluetooth connection. During testing, the batteries and other electronics for the helmet were placed inside the chest cavity of the mannequin so as not to interfere with the aerodynamics of the model cyclist.
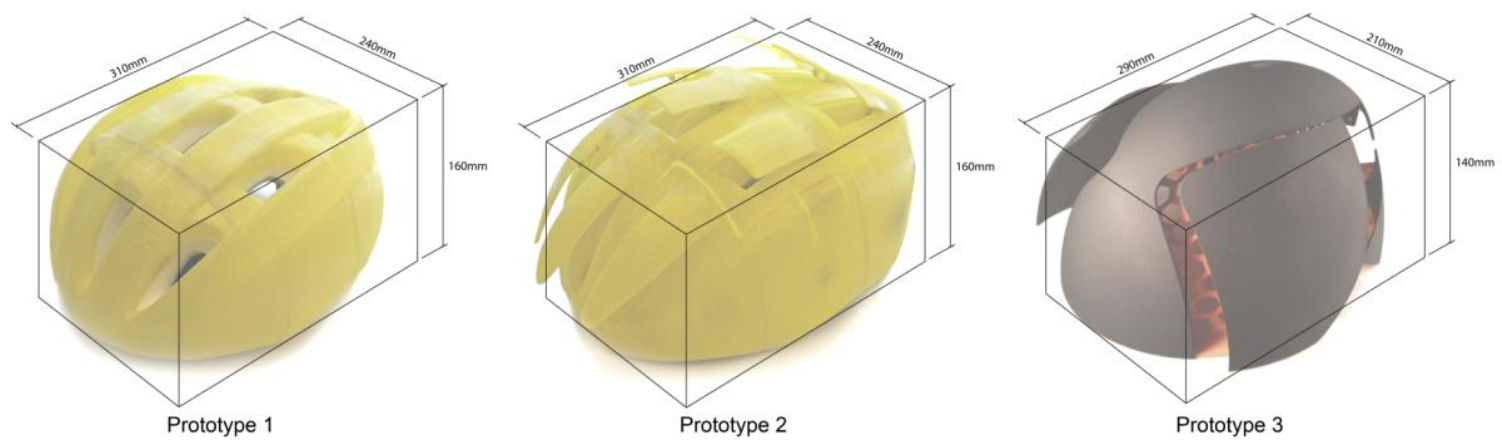

Figure 3. Overall dimensions of the three 3D printed prototypes

\section{Cycling Mannequin}

Some studies of the aerodynamic properties of bicycle helmets have used a mannequin head for testing, ${ }_{5}^{3}$ while others have used a purpose-built mannequin torso with head in a riding position. ${ }^{5,6}$ This particular study used a full-size, anthropometrically accurate mannequin representative of an adult male time-trial cyclist, sitting atop a carbon fiber racing bicycle in a road riding position similar to past studies. ${ }^{5,6}$ The mannequin, fitted with a racing skinsuit, pedaled at $80 \pm 1$ RPM for all tests so that the wheel ground speed matched wind tunnel test velocity of $44 \mathrm{~km} / \mathrm{h}$. The position of the mannequin and the cycling equipment worn by the mannequin did not vary throughout testing. Cameras were fixed around the wind tunnel circuit to capture frontal and side views of the mannequin. Images recorded by these cameras were compared between tests to determine if any movement in the mannequin's position or equipment had occurred between tests. 


\section{Wind Tunnel Facility}

A $3 / 4$ open jet wind tunnel facility located at Monash University was used for aerodynamic evaluation of the helmets used in this study. All wind tunnel experiments were performed within the $3 / 4$ open jet test section located within the return circuit of this wind tunnel as shown in Fig. 4. Wind tunnel and flow quality characteristics of the test section are shown in Table 1.

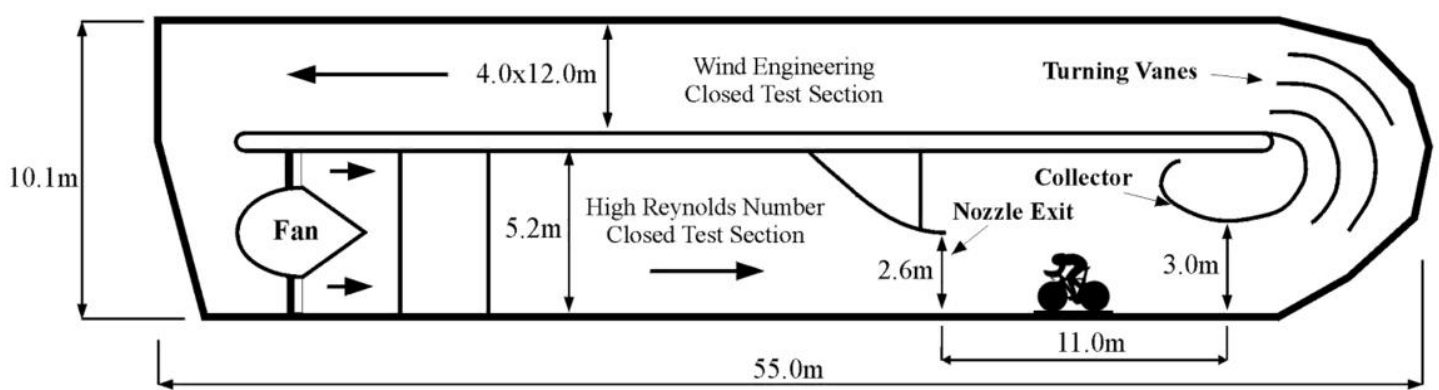

Figure 4. Monash University wind tunnel showing details of the testing location used for this study

Table 1. Wind tunnel and flow characteristics

\begin{tabular}{ll}
\hline Type & $3 / 4$ Open Jet Return \\
Jet Cross Sectional Area & $2.6 \times 4.0 \mathrm{~m}^{2}$ \\
Turbulence Intensity & $<1.6 \%$ \\
Flow Uniformity & $<1 \%$ \\
Flow Angularity & $\pm 1^{\circ}$ \\
Blockage Ratio & $<5 \%$ \\
\hline
\end{tabular}

The front and rear wheels of the fixed-gear bicycle are driven via an electric motor that powers rollers located underneath them. Due to the fixed gear design, when the rear wheel is powered, the mannequin's legs are driven around the pedal stroke.

To reduce the impact of the wind tunnel floor boundary layer on the force measurements, the mannequin and bicycle were positioned on top of a raised cantilevered platform. Struts attached to either side of the front and rear axles were used to rigidly fix the bicycle to the force balance housed underneath the wind tunnel floor. No attempt has been made to subtract aerodynamic forces acting on the struts from the measurements or correct aerodynamics forces for open-jet blockage effects. The force balance has been developed in-house at Monash University and consists of a strain gauge and floating table design utilizing air bearings.

A single test involved recording baseline measurements with no wind before and after force measurements of the cyclists so that any drift in the force measurement system over the duration of a test could be monitored and corrected for. Force measurements are taken as the mean result of three separate tests that were sampled at $500 \mathrm{~Hz}$ for 40 seconds for all helmet variations, except for the S-Works Evade which was tested five times at the start of the wind tunnel testing, with a sixth test completed at the conclusion of all testing 
to ensure wind tunnel consistency. The maximum variation in time-averaged forces for a given helmet was typically $<0.5 \%$. All aerodynamic drag measurements ' $\mathrm{D}$ ' in this study are reported as drag area measurements using Eq. (1):

$C d A=\frac{D}{\frac{1}{2} \rho U_{\infty}^{2}}$

where $\rho$ and $U_{\infty}$ represents the test section air density and test velocity respectively. The drag area is the product of the drag coefficient $(\mathrm{Cd})$ and a reference area $(\mathrm{A})$, which is typically taken as the projected frontal area of the cyclist and bicycle. The uncertainty associated with the mean calculated from repeated CdA measurements is $< \pm 0.001 \mathrm{~m}^{2}$.

\section{Results}

\section{Commercially Available Helmet Results}

The average drag area measurements for the six helmets in this study are shown in Fig. 5. Overall the Advantage and Bambino helmets had the lowest aerodynamic drag resistance when used with the visor compared to the other helmets used in this study. The CdA of these time-trial helmets was $\sim 2 \%$ lower compared to the CdA of the mannequin fitted with the S-Works Evade road helmet. Others have also shown the Giro Advantage to perform well when the aerodynamic performance of this helmet is compared with other road and time trial helmets. ${ }^{5}$

Figure 6 compares the impact of modifications made to the standard baseline helmet configurations (visor removed, vents taped) as a percentage change in CdA. Removing the visor from both the Advantage and Bambino resulted in an increase in aerodynamic drag. However, the aerodynamic performance of the Bambino helmet was far more sensitive to the removal of the visor, resulting in a $4.8 \%$ increase in $\mathrm{CdA}$ compared to the $0.8 \%$ increase for the Advantage. The increase in CdA for the Bambino suggests that this helmet was designed to only be used with the visor attached. Figure 6 also shows that closing the vents of the Advantage did not have a significant effect on its' aerodynamic performance $(\sim 0.25 \%)$. Similar studies have shown that closing the vents can reduce aerodynamic drag by as much as $12 \%$ in some helmet designs. ${ }^{6}$ However, this was not the case for the Advantage helmet, which is designed for time trial racing, where low aerodynamic resistance is the priority and the location and size of ventilation is optimized to cause minimal impact to aerodynamic performance. 


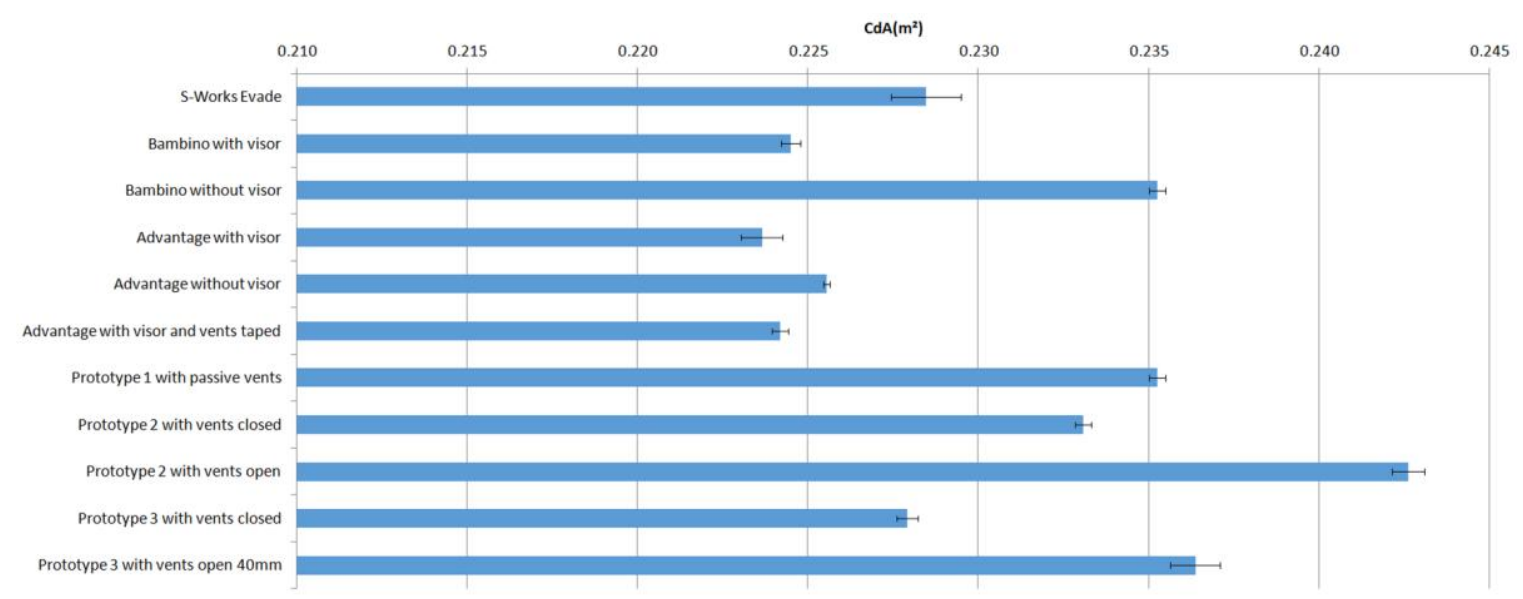

Figure 5. The average drag area $(\mathrm{CdA})$ for the six helmets with different conditions as specified at a wind speed of $44 \mathrm{~km} / \mathrm{h}$

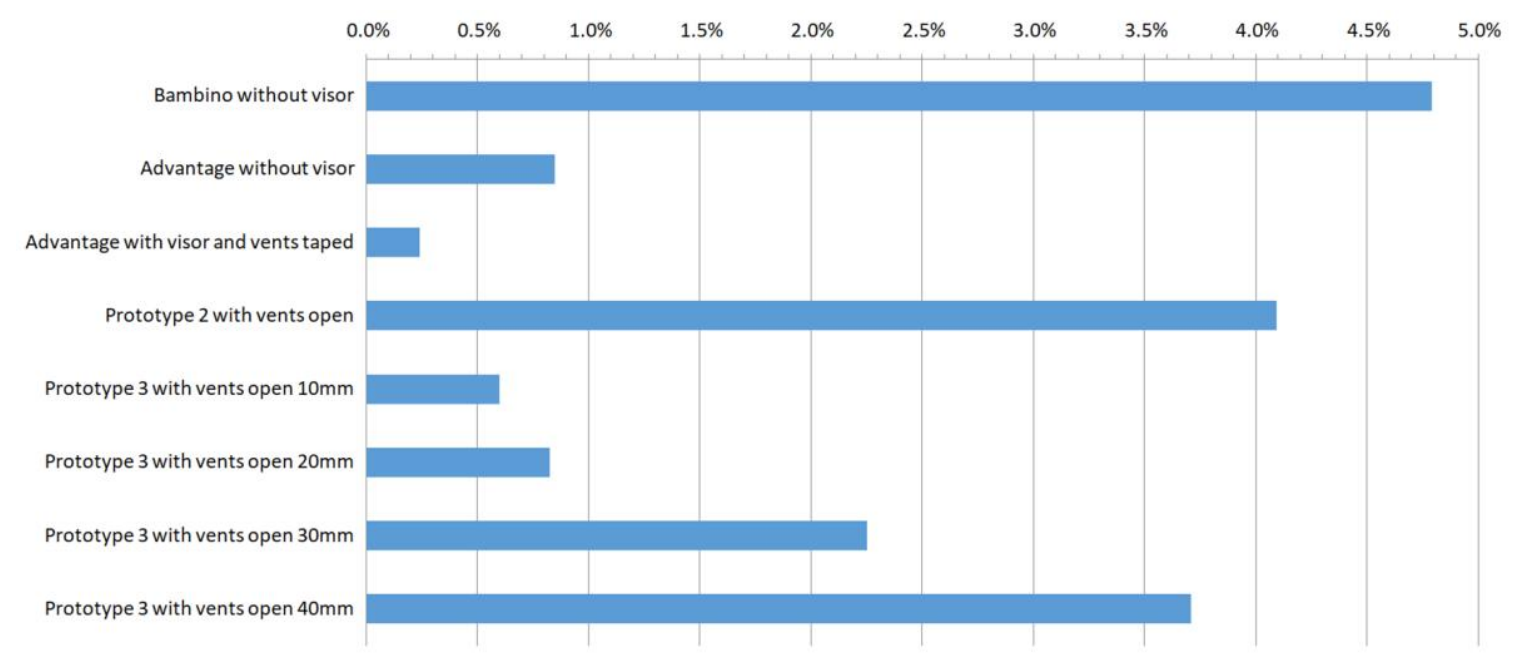

Figure 6. Comparison of helmets in different configurations

\section{Prototype Helmet Results}

Prototype 1 recorded a CdA $3.0 \%$ higher than the baseline S-Works Evade, which is not surprising given that the form of Prototype 1 was taken from a budget helmet design and the S-Works Evade is a premium helmet designed for high performance. By covering the vents, represented by Prototype 2 with vents closed, the drag area was reduced by $0.9 \%$, which would be a significant advantage for an athlete during competition. This result is similar to the Alam et al. ${ }^{6}$ experimental study which found improved aerodynamic performance with a Giro Atmos helmet when ventilation holes were taped closed. When comparing the closed and open vents of Prototype 2, a $4.1 \%$ increase in CdA occurs with the vents open. Referring to the side view of the helmet in Fig. 1e it is clear that the open vents increased projected area exposed to the flow, acting like scoops, resulting in higher CdA measurements. The ability to increase the area of the vents directly exposed to wind flow outside the normal bounds of the helmet means that there may be an increased ability to cool the head via forced convection, compared to the traditional passive vent helmet designs. This hypothesis would form part of a secondary study. 
While the extreme results (open and closed) for the electronic Prototype 3 are shown in Fig. 5, more detailed aerodynamic drag area results of the five vent positions tested in the wind tunnel are shown in Fig. 7. Here the vent positions are represented by the ratio of the projected frontal area of the vent openings to the total projected area of the helmet. The vent projected area is calculated from the size of the vent opening which was measured in the test section at wind tunnel test speed. Aerodynamic loads resulted in some movement in the vent mechanism, which was estimated to be $\pm 2.5 \mathrm{~mm}$ and is the major contributing factor to the uncertainty associated with the measurement of the vented area. Figure 8 provides a visual representation of the zones deemed to be vent areas and non-vented areas of the helmet. The total projected area of the helmet is found by summing both vented and non-vented areas.

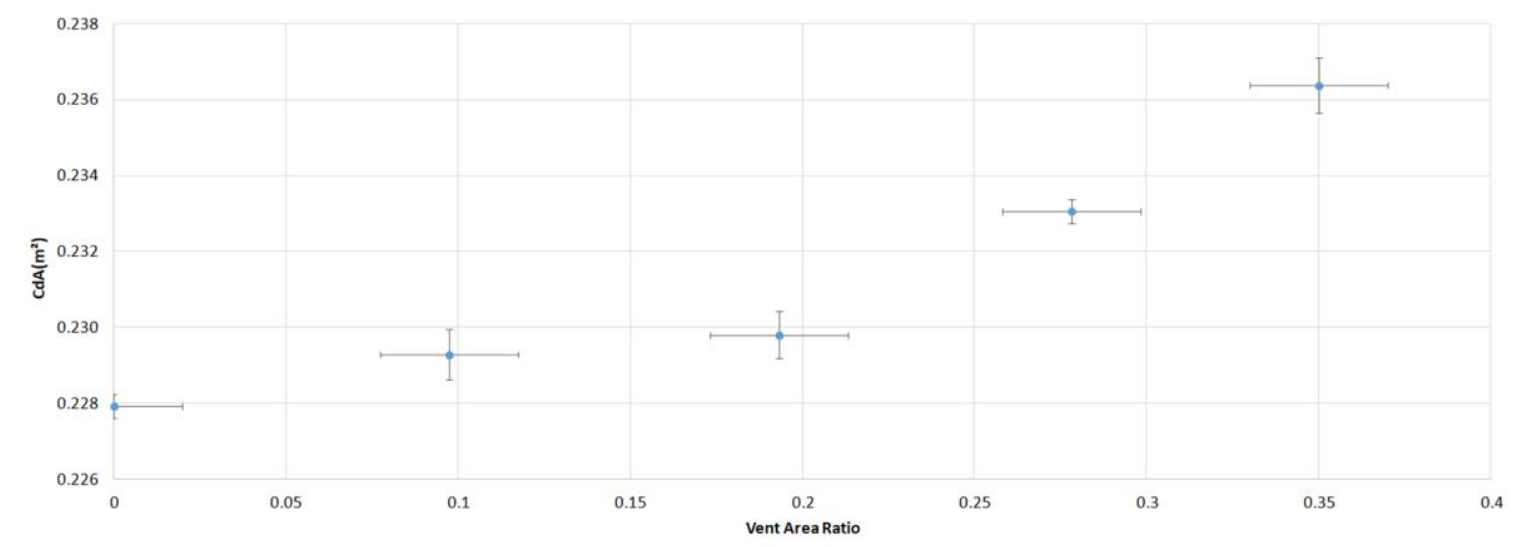

Figure 7. The average drag area (CdA) for Prototype 3 at five measured vent openings at a wind speed of $44 \mathrm{~km} / \mathrm{h}$

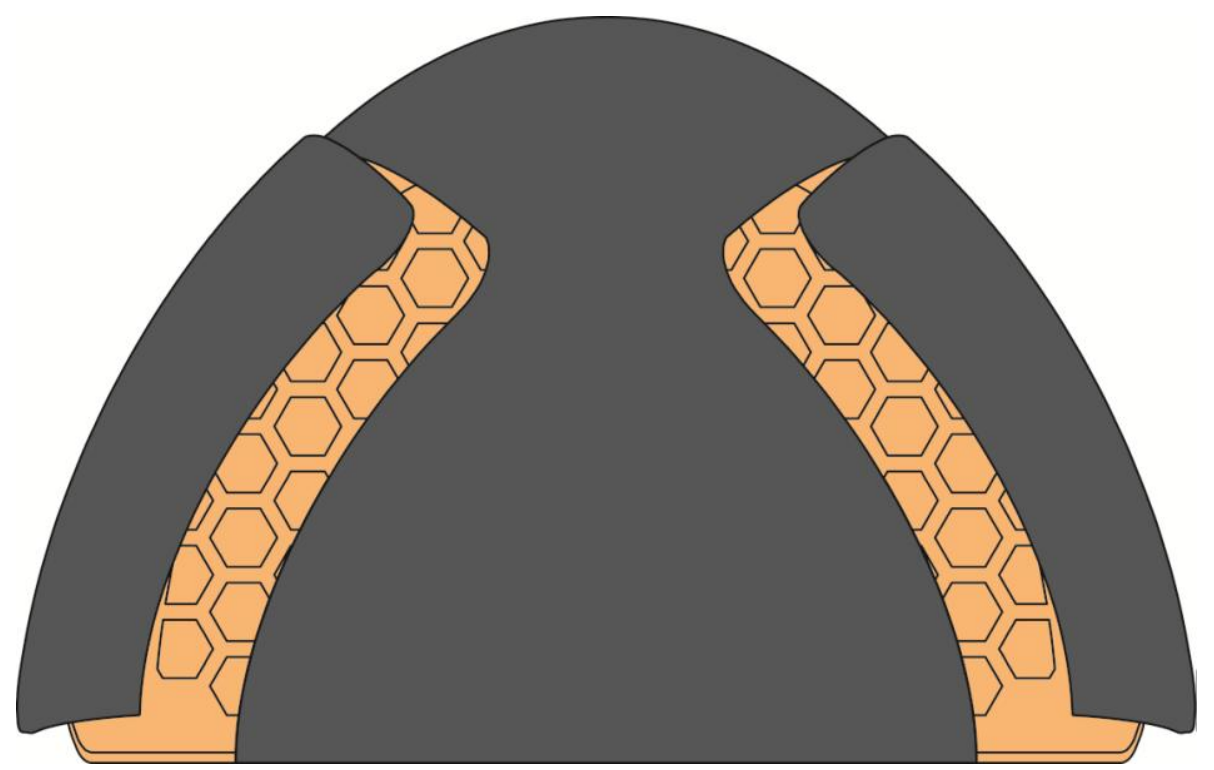

Figure 8. Diagram showing the zones calculated as Vent Area (orange with hexagon pattern) and Non-Vented Area (dark grey) for Prototype 3 with $20 \mathrm{~mm}$ vent opening 
Figure 7 shows that as the vent area ratio increases, so too does the aerodynamic drag area. A vent area ratio of 0.097 increased the drag area by $0.6 \%$ compared to the helmet in the closed position, while at the maximum ratio of 0.35 , the drag area was $3.7 \%$ greater. This is similar in magnitude to the differences recorded for open and closed vent positions for Prototype 2. In the closed position, Prototype 3 measured $0.3 \%$ less drag area than the baseline S-Works Evade.

Figure 9 highlights the change in aerodynamic drag of the test helmets in their vented and non-vented baseline states $(\triangle \mathrm{CdA})$ as a function of their vent area ratios. For a similar vent area ratio, the venting method used for Prototype 3 is superior to Prototype 2 in terms of aerodynamic efficiency. For a vented area ratio between 0.15 and 0.2 , the change in aerodynamic drag from the non-vented condition was more than three times higher for the Prototype 2 design compared to the Prototype 3 method of venting. Clearly for a given vent opening area, the design of the vents can have a significant impact on the aerodynamic forces acting on helmets.

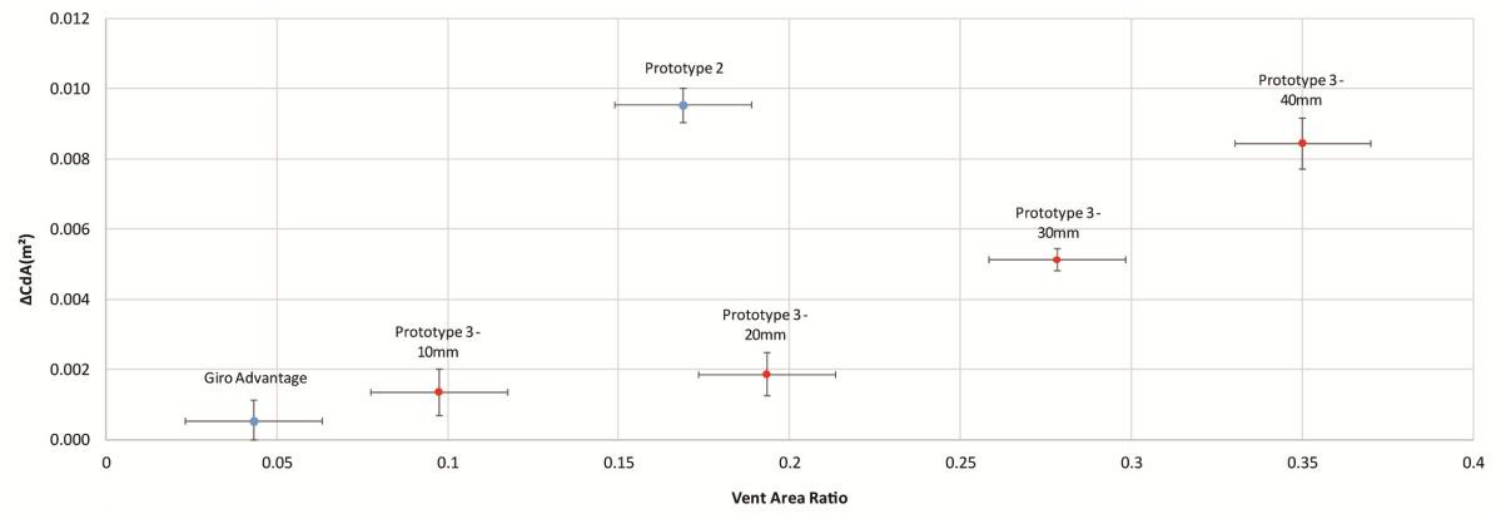

Figure 9. The change in drag area from helmets in their non-vented states $(\triangle \mathrm{CdA})$ compared to their vent area ratios

\section{Discussion}

\section{Responsive Helmets}

While a number of studies have been conducted to explore the aerodynamic properties of commercially available bicycle helmets ${ }^{3-6}$ this study includes novel prototypes that allow for the specific testing of ventilation that has been designed to be modified in a functional way. A previous study presented data about the effects of blocking ventilation holes of a Giro Atmos helmet ${ }^{6}$ however, this does not consider a practical application of this effect or the opportunity to offer degrees of cover between open and closed. As a result, the aerodynamic results for the prototype helmets in this study must be considered as part of a more complex system, rather than as simple comparisons of drag, where lower drag is typically believed to be better for cyclists.

For time-trial cycling inside a velodrome where there is a specified riding course within a closed environment, the links between aerodynamic performance, athlete comfort and 
power output will be more predictable compared to outdoor cycling activities where terrain, weather conditions, cycling speeds and the physiological cost of cycling are highly variable. At slow speeds riding uphill, aerodynamic drag forces are minimal, yet the energy exerted by the athlete is high and the need for cooling is increased. However, immediately following a hill climb, a fast decent typically occurs where studies have shown that aerodynamic efficiency is critical to athlete's speed and race-time. ${ }^{3-6}$ Both the Kask Infinity and Bell Star Pro helmets allow riders to adapt helmet properties at these times to provide better air circulation or reduce aerodynamic drag. However, riders must remember to manually change the setting each time, which could negatively affect performance if forgotten. To date, there is no peer-reviewed data regarding the effectiveness of these commercial helmet designs.

The two responsive helmet prototypes in this study are concepts aimed to automate such ventilation adjustments using electro-mechanical features. Such helmets may utilize builtin sensors or tap into existing sensors used on bicycles, such as accelerometers and power meters, to know what the rider is doing and automatically adjust settings as needed to provide optimum ventilation. A visual representation of how such a helmet may adapt is shown in Fig. 10, and while the patterns for speed and power may be somewhat simplified, they indicate how ubiquitous computing may be able to recognize patterns and respond appropriately.

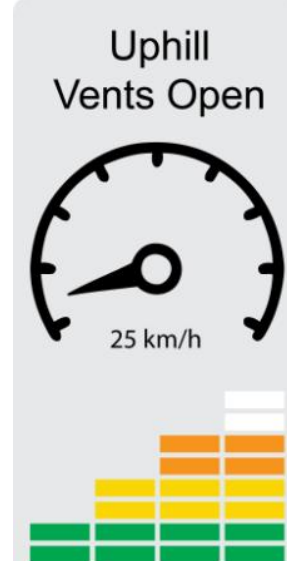

Power (watts)

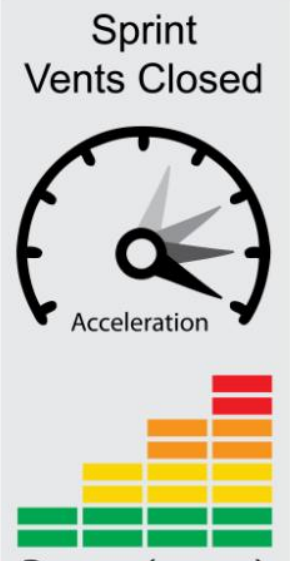

Power (watts)

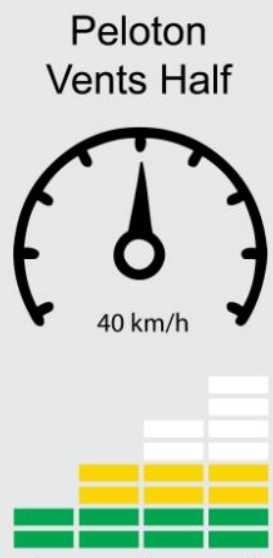

Power (watts)

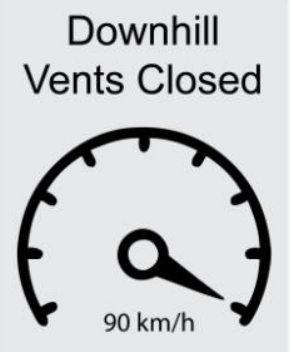

Power (watts)

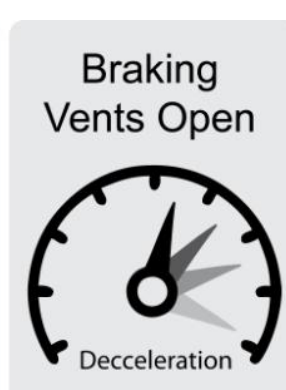

Figure 10. Common patterns during cycling that can be used to control helmet ventilation

As previously noted, the electronics of prototype helmets have been removed or simplified in order for wind tunnel testing to be performed, and the full effects of such a system have not yet been tested outside the lab. However, the data from Prototype 3 shows that aerodynamic drag forces can be varied using an electronic control mechanism. Within the confines of this experimental study, the 3D printed prototype helmets have achieved $\sim 4 \%$ variation in drag forces between their open and closed states, which may be automatically achieved in real-time using ubiquitous computing. Evidence from other sports suggests such adaptable aerodynamic performance can be utilized in many ways. For example, Formula One racing cars feature a Drag Reduction System (DRS) to assist 
with overtaking, while commercially available vehicles, like the Audi TT, exhibit an automatically adjustable spoiler, which aids in traction control at high speeds and acts as an air brake during braking. Future testing of helmet prototypes may consider such applications and are valuable considerations when understanding the results from this study as part of a more complex system, rather than straightforward comparisons of drag forces.

\section{Limitations and Future Development}

This pilot study has focused exclusively on the variations in aerodynamic drag between various helmet designs. When considering the performance of a competitive cyclist this paper only tells part of the story. The fluid mechanisms leading to variations in aerodynamic properties of the helmet and the cyclist clearly require further investigation. This pilot study has demonstrated the potential for the geometric properties of the helmet to be tuned to optimize rider performance criteria for various cycling scenarios. However, a detailed understanding of the aerodynamics of helmet design and the flow interactions that occur between the helmet geometry and the flow over a rider, where the majority of the pressure drag acting originates, is lacking.

In addition to the aerodynamics, the thermal properties of the helmet and the ability of an athlete to regulate body temperature is impacted by helmet ventilation design. Future testing will allow a more comprehensive assessment of responsive bicycle helmet abilities to regulate thermal and aerodynamic properties by modifying ventilation openings electronically. The study by Alam et al. ${ }^{6}$ demonstrated a $\sim 1.2^{\circ} \mathrm{C}$ increase in head temperature at a wind speed of $30 \mathrm{~km} / \mathrm{h}$ when a helmet had some of its vents taped closed compared to the original helmet. However, this temperature difference disappeared at wind speeds of $\sim 45 \mathrm{~km} / \mathrm{h}$ and greater. The effect at speeds less than 30 $\mathrm{km} / \mathrm{h}$ is unknown as no data was collected at these lower wind speeds. Similar insights are needed for responsive helmets to map the changes in thermal properties as vents open, close and change form. Future studies will also consider the forces on the cyclist's neck as ventilation opens.

Prototypes will also need to consider equipment regulations with helmets being compulsory under Article 1.3.031 of the 'Clarification Guide of the UCI Technical Regulation. ${ }^{\text {'9 }}$ While Prototype 3 highlights potential opportunities for a cyclist in terms of aerodynamic performance, UCI Article 1.3.031 states that "the use of mechanical or electronic systems in or on the helmet is also prohibited." "Furthermore, Article 1.3.033 states that "equipment (helmets, shoes, jerseys, shorts, etc.) worn by the rider may not be adapted to serve any other purpose apart from that of clothing or safety by the addition or incorporation of mechanical or electronic systems." "While rules frequently change, significant research is needed to validate the safety of electromechanical helmets and the benefits to athlete performance and health.

\section{Conclusion}


This experimental research provides new wind tunnel data regarding the aerodynamic properties of various bicycle helmets and venting systems, including prototypes for new forms of responsive helmets. Both commercially available and prototype helmets were used to investigate the impact that different venting methods have on aerodynamic performance. While a commercially available Giro Advantage time trial helmet recorded the lowest drag forces, blocking its vents was found to negatively affect aerodynamic performance. The variation on aerodynamic drag between closed and open vent conditions was also compared between the prototype helmets used in this study. Results showed that the aerodynamic performance of the helmets was dependent not only on the size of the vents, but also on the design and method used for ventilation. The prototype helmets demonstrate the potential to automate control of adjustable ventilation to modify drag characteristics for a variety of racing scenarios, to optimize the aerodynamic and cooling properties using embedded computing capabilities. Future studies are in development to determine the link between variable helmet ventilation systems and athlete performance measures, including athlete cooling and aerodynamic efficiency.

\section{Declaration of Conflicting Interests}

The author(s) declared no potential conflicts of interest with respect to the research, authorship and/or publication of this article.

\section{Funding}

The author(s) received no financial support for the research, authorship and/or publication of this article.

\section{References}

1. Epstein D. David Epstein: Are athletes really getting faster, better, stronger?, http://www.ted.com/talks/david_epstein_are_athletes_really_getting_faster_better_strong er/ (2014, accessed 16 May 2014).

2. Hamilton R. Racing Machines. Le Tour de France: The Greatest Race in Cycling History. West Sussex, UK: Summersdale Publishers Ltd, 2013.

3. Alam F, Subic A and Watkins S. A Study of Aerodynamic Drag and Thermal Efficiency of a Series of Bicycle Helmets. In: Moritz EF and Haake S (eds) The Engineering of Sport 6: Volume 1: Developments for Sports. New York, NY: Springer New York, 2006, pp.127-131.

4. Alam F, Subic A, Akbarzadeh A, et al. Effects of venting geometry on thermal comfort and aerodynamic efficiency of bicycle helmets. In: F. Fuss AS, S. Ujihashi (ed) The Impact of Technology on Sport II. Netherlands: Taylor and Francis Group 2008, pp.773-780.

5. Chowdhury $\mathrm{H}$ and Alam F. An experimental study on aerodynamic performance of time trial bicycle helmets. Sports Eng 2014; 17: 165-170. DOI: 10.1007/s12283-0140151-5. 
6. Alam F, Chowdhury H, Elmir Z, et al. An experimental study of thermal comfort and aerodynamic efficiency of recreational and racing bicycle helmets. Procedia Engineering 2010; 2: 2413-2418. DOI: http://dx.doi.org/10.1016/j.proeng.2010.04.008.

7. Weiser M. The Computer for the 21st Century. Scientific American 1991; 265: 94-110.

8. Novak J and Loy J. Digital Technologies and 4D Customized Design:

Challenging Conventions with Responsive Design. In: Bryan VC, Musgrove AT and Powers JR (eds) Handbook of Research on Human Development in the Digital Age. Hershey, PA, USA: IGI Global, 2017, pp.403-426.

9. Internationale UC. Clarification Guide of the UCI Technical Regulation. In: Internationale UC, (ed.). 1/1/2017 ed.: Union Cycliste Internationale, 2017, p. 47. 